\title{
Picometer-Stable Hexagonal Optical Bench to Verify LISA Phase Extraction Linearity and Precision
}

\author{
Thomas S. Schwarze, Germán Fernández Barranco, Daniel Penkert, \\ Marina Kaufer, ${ }^{\dagger}$ Oliver Gerberding, and Gerhard Heinzel \\ Max Planck Institute for Gravitational Physics (Albert Einstein Institute), Callinstrasse 38, 30167 Hannover, Germany \\ and Leibniz Universität Hannover, Institut für Gravitationsphysik, Callinstrasse 38, 30167 Hannover, Germany
}

(Received 5 October 2018; revised manuscript received 6 December 2018; published 28 February 2019)

\begin{abstract}
The Laser Interferometer Space Antenna (LISA) and its metrology chain have to fulfill stringent performance requirements to enable the space-based detection of gravitational waves. This implies the necessity of performance verification methods. In particular, the extraction of the interferometric phase, implemented by a phasemeter, needs to be probed for linearity and phase noise contributions. This Letter reports on a hexagonal quasimonolithic optical bench implementing a three-signal test for this purpose. Its characterization as sufficiently stable down to picometer levels is presented as well as its usage for a benchmark phasemeter performance measurement under LISA conditions. These results make it a candidate for the core of a LISA metrology verification facility.
\end{abstract}

DOI: 10.1103/PhysRevLett.122.081104

Introduction.-The first detections of gravitational waves by the Laser Interferometer Gravitational-Wave Observatory (LIGO) [1] and Virgo [2] have opened the window for gravitational wave astronomy in the $\mathrm{Hz}$ and $\mathrm{kHz}$ range. Avoiding limitations by seismic and gravity gradient noise, the Laser Interferometer Space Antenna (LISA) $[3,4]$ will offer revolutionary science with sources only detectable in the $\mathrm{mHz}$ regime. Important examples are extreme mass ratio inspirals for strong-gravity tests of general relativity or massive black hole binaries at red shifts up to 20 to study their early formation [4].

LISA consists of three spacecraft (SC) forming a triangular constellation with 2.5 million $\mathrm{km}$ arm lengths. It will measure the displacement between free-falling test masses (TMs) by means of heterodyne laser interferometry. The latter is split into local TM-SC and remote SC-SC displacement measurements. The targeted band of $0.1 \mathrm{mHz}$ to $1 \mathrm{~Hz}$ will be limited above $3 \mathrm{mHz}$ by the optical metrology $(10 \mathrm{pm} / \sqrt{\mathrm{Hz}}$; with $4.7 \mathrm{pm} / \sqrt{\mathrm{Hz}}$ shot noise), and below by TM stray accelerations $\left(3 \mathrm{fm} \mathrm{s}^{-2} / \sqrt{\mathrm{Hz}}\right)$.

The LISA Pathfinder mission impressively showed the feasibility of the stringent stray acceleration target [5] as well as local interferometry with $35 \mathrm{fm} / \sqrt{\mathrm{Hz}}$ precision [6]. In addition, the recently launched satellite geodesy mission Gravity Recovery and Climate Experiment Follow-On (GRACE-FO) carries the first SC-SC interferometer with

Published by the American Physical Society under the terms of the Creative Commons Attribution 4.0 International license. Further distribution of this work must maintain attribution to the author(s) and the published article's title, journal citation, and DOI.
$80 \mathrm{~nm} / \sqrt{\mathrm{Hz}}$ targeted precision [7]. Like a LISA SC-SC interferometer, it operates at weak-light levels $(\sim 100 \mathrm{pW})$. However, LISA is more demanding in terms of precision and in the aspects explained in the following.

Most importantly, each LISA SC-SC interferometer will exhibit coupling of the full laser frequency noise due to the SC distances acting as huge interferometer arm mismatches. Mitigation of this otherwise overwhelming noise coupling will be performed by a technique called time-delay interferometry (TDI) [8,9]. Primarily, it time shifts and combines SC-SC measurements throughout the constellation in postprocessing to cancel multiple but delayed occurrences of laser frequency noise. However, as TDI is performed in postprocessing, the interferometer phase extraction by a phasemeter has to conserve the essential displacement information hidden in the much stronger laser frequency noise. Therefore, linearity over a high dynamic range is needed while maintaining phase fidelity. The latter is defined by the phasemeter (singlechannel) noise contribution requirement of $1 \mu$ cycle $/ \sqrt{\mathrm{Hz}}$ or $2 \pi \mu \mathrm{rad} / \sqrt{\mathrm{Hz}}(\sim 1 \mathrm{pm} / \sqrt{\mathrm{Hz}}$ for a $1064 \mathrm{~nm}$ laser $)$ down to $3 \mathrm{mHz}$ with a relaxation due to the dominating TM stray acceleration below. Hence, with expected master laser stabilities [10], the required dynamic range in orders of magnitude is 8 at $1 \mathrm{~Hz}$ and rises to 10 below $3 \mathrm{mHz}$.

Also, the phasemeter needs to cope with heterodyne frequencies of 5-25 MHz and change rates up to $20 \mathrm{~Hz} / \mathrm{s}$. These values are determined by the expected Doppler shifts due to SC motion as well as by offsets intentionally applied for constellation-wide frequency planning.

As a consequence, the verification of the described phasemeter requirements is crucial to complete the 
demonstration of the LISA metrology. A frequently utilized verification scheme is a split test, which is based on a differential measurement of identical signals. In the past, various phasemeters have been reported to show phase noise performances [11-13] below $1 \mu$ cycle $/ \sqrt{\mathrm{Hz}}$ in split tests. Yet, particularly for LISA, a more elaborate verification scheme is needed, e.g., a three-signal test, first mentioned in Ref. [13]. Applying nonidentical signals to the phasemeter channels, it reveals noise sources that in a split test cancel out undetected as common mode. Most importantly, it also allows the test of phase extraction linearity, as shown later. However, conducted electrical and optical three-signal tests so far are at least an order of magnitude above the required precision, mainly limited by test bed noise [12,14]. Digital three-signal tests $[13,15]$ verified performance of the digital phasemeter core (presented later), while by definition they cannot test the typically limiting analog front end.

This Letter reports on a hexagonal quasimonolithic optical bench implementing an optical three-signal test. It shows sufficient stability for LISA phasemeter linearity and precision verifications. After the experimental setup, measurements proving the capabilities of the test bed together with a benchmark performance test of a LISA phasemeter will be shown.

Experimental setup.-First, the optical three-signal scheme will be described. Here, phase is defined as instantaneous phase $\varphi(t)$ of a harmonic signal $x(t)$ with amplitude $a$. It is closely related to the instantaneous frequency $f(t)$ and the carrier frequency offset $\bar{f}$ :

$$
x(t)=a \sin (\varphi(t)), \quad d \varphi(t) / d t=2 \pi[f(t)+\bar{f}] .
$$

For testing, three initial signals (electric fields of laser beams exhibiting unequal optical frequency offsets) with phases $\varphi_{1}, \varphi_{2}, \varphi_{3}$ are interfered pairwise. Each resulting laser beam intensity contains two mixed initial signals which generate a beat note. The beat note signals can exhibit unequal $\mathrm{MHz}$ frequency offsets, called heterodyne frequencies $\bar{f}_{a} \neq \bar{f}_{b} \neq \bar{f}_{c}$, and phases $\varphi_{a}, \varphi_{b}, \varphi_{c}$ :

$$
\varphi_{a}=\varphi_{1}-\varphi_{2}, \quad \varphi_{b}=\varphi_{2}-\varphi_{3}, \quad \varphi_{c}=\varphi_{3}-\varphi_{1} .
$$

After conversion to voltages by photoreceivers, three phasemeter channels extract the measured phases $\varphi_{a}^{\prime}, \varphi_{b}^{\prime}$, and $\varphi_{c}^{\prime}$ from the beat notes. With the operator $\mathcal{E}$ denoting the phase fidelity of the extraction, it can be written

$\varphi_{a}^{\prime}=\mathcal{E}\left(\varphi_{1}-\varphi_{2}\right), \quad \varphi_{b}^{\prime}=\mathcal{E}\left(\varphi_{2}-\varphi_{3}\right), \quad \varphi_{c}^{\prime}=\mathcal{E}\left(\varphi_{3}-\varphi_{1}\right)$.

Finally, the three measured phases are combined in postprocessing to form the three-signal measurement

$$
\varphi_{0}=\varphi_{a}^{\prime}+\varphi_{b}^{\prime}+\varphi_{c}^{\prime} \stackrel{?}{\sim} 0,
$$

which is the main measurement and in which the initial phases ideally cancel. It includes the phase noise contribution of the phasemeter while being sensitive to nonlinearities: if $\mathcal{E}$ is nonlinear, which means the condition $\mathcal{E}\left(\varphi_{1}-\varphi_{2}\right)=\mathcal{E}\left(\varphi_{1}\right)-\mathcal{E}\left(\varphi_{2}\right)$ does not hold, the generic initial phases will not cancel pairwise in $\varphi_{0}$. The same is true for nonlinear effects due to the unequal heterodyne frequencies. Additionally, the ratio between $\varphi_{0}$ and the single channel inputs $\varphi_{a-c}^{\prime}$ gives a direct estimate of the phasemeter dynamic range.

Here, the scheme is implemented using a hexagonal quasimonolithic bench as test bed core. The complete test bed is divided into three conceptual parts.

The first part is a laser preparation bench. It provides the initial signals with phases $\varphi_{1-3}$ using three $1064 \mathrm{~nm}$ lasers (Mephisto by Coherent, $500 \mathrm{~mW}$ ). As their pairwise combinations are supposed to generate beat notes with heterodyne frequencies of $5-25 \mathrm{MHz}$, their frequency relation must be well defined. This is achieved by two digital control loops locking the frequencies of two slave lasers to one master laser. The loop reference signal then sets the desired heterodyne frequencies and can be utilized to add artificial LISA-like frequency noise to $\varphi_{a-c}$ to test the required dynamic range.

The second part is the LISA phasemeter under test here. It was developed within a European Space Agency (ESA) contract [16] by a European consortium. It is based on parallel analog-to-digital converter channels $(80 \mathrm{MHz})$ connected to field-programmable gate arrays (FPGAs) implementing digital phase-locked loops (DPLLs). The frequencies tracked by the DPLLs are downsampled and converted to phase in postprocessing. While the performance of the DPLL serving as phasemeter core was verified in the aforementioned digital three-signal test [15], it was also shown that the phasemeter utilized here fulfills the LISA requirements in electrical split tests [11].

Finally, the core of the experiment is the aforementioned quasimonolithic optical bench. It was designed using the software tool IfoCAD [17] and enables the stable splitting and interference of the initial signals according to the threesignal scheme. Figure 1 shows a schematic of the bench. It consists of a thick Zerodur baseplate $(200 \times 200 \times 25 \mathrm{~mm})$ carrying three fiber injector optical subassemblies (FIOSs) for the injection of the prepared laser light and six wedged beam splitters (roughly 50:50 split ratio) placed in a hexagonal layout. Three of the beam splitters act as a first stage where each of the injected beams (carrying the phases $\left.\varphi_{1-3}\right)$ is divided into two. In a second stage, each divided beam is interfered pairwise with the output of another dividing beam splitter, forming three interferometers in total. Finally, the interferometer outputs at the second stage beam splitters yield the three beat notes with heterodyne frequencies $\bar{f}_{a-c}$ and phases $\varphi_{a-c}$ as well as three complementary versions with phases $\varphi_{a-c}^{*}$ (shifted by $\pi$ ). The six output beat notes are captured by photoreceivers and are 


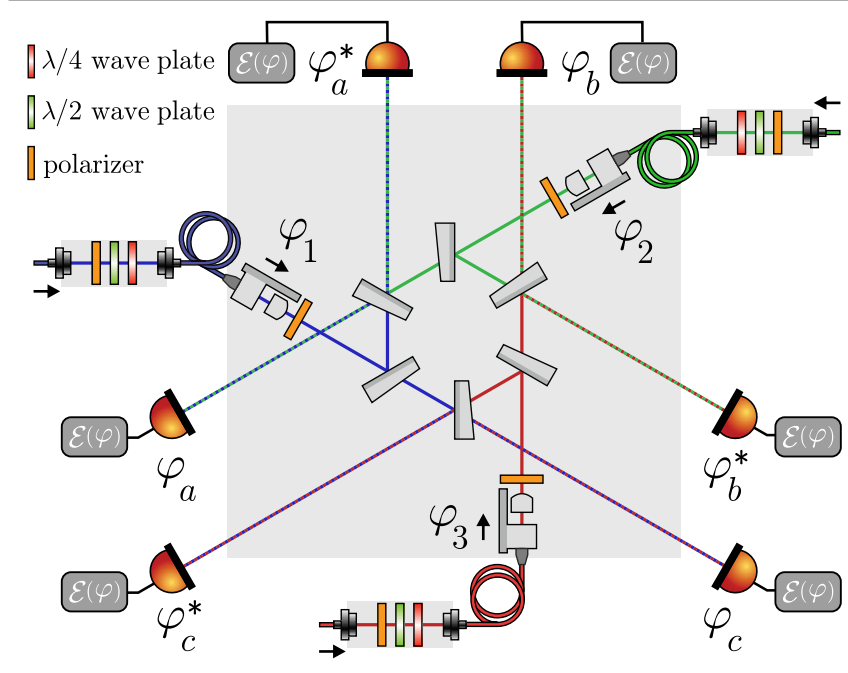

FIG. 1. Schematic of the hexagonal quasimonolithic optical bench implementing a three-signal linearity test. Complementary interferometer outputs are utilized for diagnostic $\pi$ pretests.

subsequently sampled by the phasemeter. The photoreceivers, designed for the test bed using off-the-shelf components, comprise InGaAs photodiodes $(0.5 \mathrm{~mm}$ in diameter, $\sim 1 \mathrm{~mW}$ incident power) and transimpedance amplifiers with a single operational amplifier. At each output beam splitter, one of the complementary signals can be picked for the three-signal test. Also, both signals' phases can be averaged for balanced detection or be subtracted for an optical split test with a $\pi$ phase shift, named $\pi$ measurement. The latter exhibits shortcomings similar to the split tests described above, and is considered a diagnostic pretest. It can reveal noise sources it has in common with the three-signal test, e.g., some types of stray light. A subset of these can be canceled with balanced detection.

While not being the only topology possible for a threesignal scheme, the presented approach is well-suited concerning two critical aspects. One is the phase noise coupling after the split of the initial phases. In general, this kind of noise $\varphi_{N}$ is not common and acts in one phasemeter channel only, as, e.g., $\mathcal{E}\left(\varphi_{1}-\varphi_{2}+\varphi_{N}\right)$ shows. Hence, it cannot be distinguished from phasemeter noise and limits the test bed. In the hexagonal bench, this noise is primarily determined by the displacement stability between the first and second stage beam splitters. The hexagonal configuration allows a compact and symmetric implementation of the interferometers, hence, lowering the noncommon-mode displacement fluctuations caused by thermal expansion and mechanical distortions. For further mitigation of those effects, the thick Zerodur baseplate serves as thermal bulk with low thermal expansion coefficient while the fused silica beam splitters are attached via hydroxide-catalysis bonding [18] and optical contacting. Because of its repeatability, the latter method was chosen for the placement of the second stage beam splitters which are decisive for proper contrast.
A second critical aspect of the bench is the static mismatch of the two displacements between any first stage beam splitter and its successive second stage beam splitters. In general, such a mismatch acts as unequal interferometer arms and hence leads to the coupling of single laser frequency noise limiting the test bed. This unwanted noise should not be confused with the controlled differential laser frequency noise in $\varphi_{a-c}$ used to mimic the master laser frequency noise in LISA and which is meant to cancel out. Nevertheless, the symmetric layout of the hexagonal interferometers allows the reduction of the coupling by matching the static displacements within assembly tolerances. A maximum displacement mismatch of $\sim 200 \mu \mathrm{m}$ is assumed.

As an amendment to the stable hexagonal interferometer design, the aforementioned FIOSs were added to minimize thermally induced angular jitter of the input beams. The FIOSs consist of glued fused silica components and are based on adaptations of earlier designs [19]. The minimization of the angular jitter is desirable as it couples into displacement noise via the wedged beam splitters. The latter in turn were chosen to achieve the angular separation of desired beams and ghost beams reflected from secondary surfaces.

The optical bench, together with auxiliary optics and photoreceivers, is placed in a vacuum chamber. For proper operation, a moderate vacuum (roughly $<10 \mathrm{mbar}$ ) is required, primarily to avoid optical path length fluctuations caused by residual air. A fiber interface connects the external laser preparation to the optical bench.

Another essential aspect for the operation of the test bed is a proper polarization control. Ideally, all beams in the interferometer should exhibit the same polarization axis. Mismatches lead to secondary parasitic interferometers in the orthogonal axis. The closest approach to the ideal case was achieved with laminated thin-film polarizers (extinction ratio $1: 10^{7}$ ) placed right after the FIOSs and with their transmissive axis set to the bench surface normal as a common reference. The polarization cleanliness was improved further by controlling the input of the polarizationmaintaining FIOS fibers. For that purpose, pairs of $\lambda / 2$ - and $\lambda / 4$-wave plates optimally match the fiber input beam polarization to the fiber slow axis. This also attenuates indirect coupling chains, e.g., fiber polarization fluctuations to pointing jitter to phase front jitter to phase noise.

To summarize, the presented setup aims to minimize all test bed noise indistinguishable from the noise contribution of the phasemeter. The residual noise floor gives an upper bound for the phasemeter performance in a three-signal test, while the digital laser control can create LISA-like phase input conditions.

An important extension will be the utilization of three separate phasemeters with independent clocks [20], which would allow testing of LISA SC-SC features like clock tone transfer, ranging and data transfer as well as postprocessing 


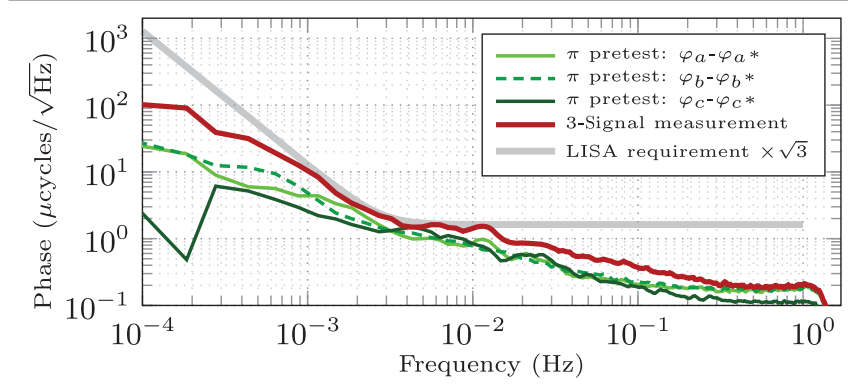

FIG. 2. Measurement ( $3 \mathrm{~h})$ with moderate phase noise input conditions. The three-signal combination (red) fulfills the LISA requirement scaled for three signals. Its fundamental noise can be found in the $\pi$ pretests (green), suggesting sufficient stability of the hexagonal optical bench itself.

techniques like interpolation, clock synchronization, and clock noise removal for TDI. This way, the setup can provide realistic data to support scientists developing the LISA data processing and analysis. Also, the attenuation of a single beam to $100 \mathrm{pW}$ will allow proper testing of dedicated LISA photoreceivers.

Results.-In the following, three measurements carried out with the described setup are presented. One was conducted with low heterodyne frequencies $(5.8,3.01$, and $2.79 \mathrm{MHz}$ ) and input phase noise of 0.04 cycles $/ \sqrt{\mathrm{Hz}}$ at $1 \mathrm{~Hz}$. These values allowed excluding from the performance assessment effects such as dynamic range limitations or noise caused by high carrier frequencies. The resulting spectral densities [21,22] are shown in Fig. 2. The three-signal performance (red line, best pick from each of the complementary output pairs) satisfies the LISA phase extraction requirement scaled for three uncorrelated signals. Above $1 \mathrm{mHz}$, the $\pi$ pretests (green lines) at the three interferometer outputs show a similar noise shape and magnitude, while balanced detections (not shown) did not yield a significant improvement. Hence it is assumed that, in this frequency band, the dominant noise source in the three-signal test is the same as in the $\pi$ pretests. Vanishing as common mode in the latter, instability of the optical bench can be excluded. Instead, residual polarization mismatches are considered as the main limitation candidate, as small changes in the polarizer alignments easily spoil both $\pi$ and three-signal tests. This suggests that the test bed performance, not limited by the hexagonal optical bench itself, could be improved further.

More measurements were conducted with LISA-like input conditions as specified in the following. The results are shown in Fig. 3. For the first three-signal measurement (red solid line), the heterodyne frequencies were set to 24.9, 18.1 , and $6.8 \mathrm{MHz}$, while the single input phases (top lines) were generated to resemble a LISA-like signal shape with instantaneous frequency noise of $450 \mathrm{~Hz} / \sqrt{\mathrm{Hz}}$ at $1 \mathrm{~Hz}$ $(\sim 70$ cycles $/ \sqrt{\mathrm{Hz}})$ and a $1 / f$ behavior dominating below $3 \mathrm{mHz}$. With the shown three-signal performance, this corresponds to a dynamic range of 8,10 , and 11 orders of

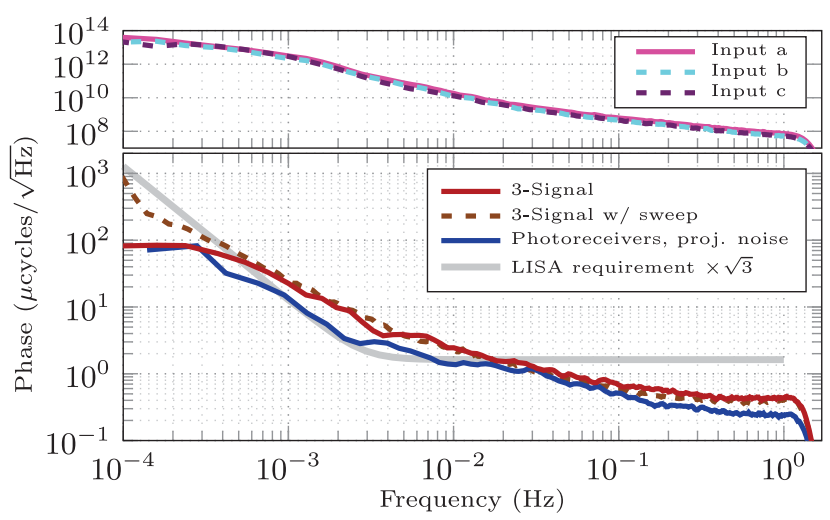

FIG. 3. Measurements with LISA-like input phase noise and heterodyne frequencies. A dynamic range of 8-11 orders of magnitude can be computed from the three input signals (top) and the three-signal combinations (red: fixed heterodyne frequencies, $3 \mathrm{~h}$; brown dashed: heterodyne frequency sweep over $90 \mathrm{~h}$ ). The LISA three-signal requirement was fulfilled except between 0.4-20 $\mathrm{mHz}$, with the photoreceivers being a major noise contributor (noise projection in blue).

magnitude at $1 \mathrm{~Hz}, 3 \mathrm{mHz}$, and below $1 \mathrm{mHz}$, respectively. This is illustrated in Fig. 4 which shows time series of the single input phase fluctuations and their combination in a drastically reduced scale (right side). The performance satisfied the LISA phase extraction requirement except in the range of $0.4-20 \mathrm{mHz}$ where a violation by a factor 3 occurs. Nevertheless, when summed quadratically with other noise sources for the overall $10 \mathrm{pm} / \sqrt{\mathrm{Hz}}$ budget, it is still not significantly limiting. A major contribution to the higher noise level compared to the measurement with moderate input conditions was traced back to the utilized photoreceivers. At the required precision levels, they show a heterodyne-frequency-dependent noise behavior and thus lead to excess noise when operated at the upper end of the LISA heterodyne frequency band. Isolated differential measurements between pairs of photoreceivers were conducted for a noise projection (Fig. 3, blue line). This includes a higher noise contribution by the phasemeter itself, which shows a similar, but weaker heterodynefrequency dependence.

A second long-term three-signal measurement (Fig. 3, brown dashed line) was conducted with similar input

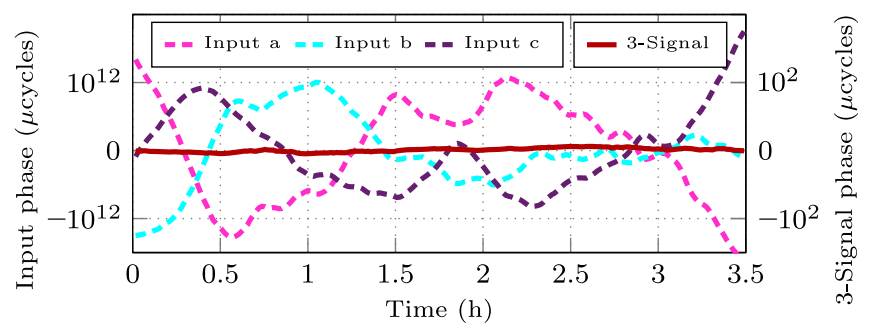

FIG. 4. Time series of input phase fluctuations and resulting three-signal combination, illustrating the high dynamic range essential for TDI. 
phase noise as in the prior measurement but with dynamic heterodyne frequencies. More specifically, sweeps from 24.9-5.0 MHz, 18.1-3.0 MHz, and 6.8-2.0 MHz were applied within a time frame of $90 \mathrm{~h}$. This corresponds to a maximum sweep rate of $\sim 61 \mathrm{~Hz} / \mathrm{s}$. Compared to the prior measurement, the performance shows no significant deterioration except a stronger low-frequency drift.

Discussion and conclusions.-The measurement presented in Fig. 2 shows that the hexagonal optical bench provides sufficient stability down to LISA-like picometer levels and a static displacement matching that allowed the use of a free-running master laser. These properties in turn enabled the measurement to be the first optical three-signal linearity test with $\mathrm{MHz}$ signals and $\mu$ cycle $/ \sqrt{\mathrm{Hz}}$ precision. Additionally, the $\pi$ pretests suggest that the hexagonal bench stability is currently not limiting above $1 \mathrm{mHz}$ and that the performance could be improved further.

Nevertheless, the test bed already enabled a benchmark linearity test of a phasemeter with LISA-like dynamic range and heterodyne frequencies (Fig. 3). The required phase extraction performance could be verified in most of the frequency band, with the phasemeter likely not being limiting in the current state. Instead, a major noise contributor are the photoreceivers. Yet, they are not considered a show stopper and will be investigated further.

Comparable state-of-the-art test beds $[14,23]$ were able to show similar dynamic ranges, however with single-digit $\mathrm{MHz}$ frequencies and most importantly with precision levels more than an order of magnitude above the ones demonstrated in this Letter.

The shown results suggest the utilization of the hexagonal optical bench test bed as a facility for the verification of future iterations of the LISA phasemeter, including engineering or flight models. As mentioned, the test bed can easily be extended to probe other important features of the LISA metrology chain and to support LISA data processing and analysis.

Besides this extension, future work will include efforts to tackle the noise sources assumed to be limiting, like polarization and photoreceivers, to reduce the test bed noise floor further.

To conclude, the LISA phasemeter in particular and the LISA metrology chain in general are crucial for the successful detection of gravitational waves in space. Stringent requirements are imposed on these components, making verification a challenge. The measurements presented here show that the hexagonal optical bench provides the capability to face this challenge successfully.

The authors acknowledge financial support by the European Space Agency (ESA) (22331/09/NL/HB, $16238 / 10 / \mathrm{NL} / \mathrm{HB})$, the German Aerospace Center (DLR) (50OQ0601, 50OQ1301) and the Deutsche Forschungsgemeinschaft (DFG) Sonderforschungsbereich 1128 Relativistic Geodesy and Gravimetry with Quantum Sensors (geo-Q). *thomas.schwarze@aei.mpg.de

Present address: SpaceTech GmbH (STI), Seelbachstrasse 13, 88090 Immenstaad am Bodensee, Germany.

[1] B. P. Abbott et al. (LIGO Scientific and Virgo Collaborations), Observation of Gravitational Waves from a Binary Black Hole Merger, Phys. Rev. Lett. 116, 061102 (2016).

[2] B. P. Abbott et al. (LIGO Scientific and Virgo Collaborations), GW170814: A Three-Detector Observation of Gravitational Waves from a Binary Black Hole Coalescence, Phys. Rev. Lett. 119, 141101 (2017).

[3] P. Bender et al. (LISA Study Team), LISA Pre-Phase A report, MPQ 233 version 2.08, 1998, https://lisa.nasa.gov/ archive2011/Documentation/ppa2.08.pdf.

[4] K. Danzmann et al., LISA Laser Interferometer Space Antenna, response to ESA L3 mission call, 2017, https:// www.elisascience.org/files/publications/ LISA_L3_20170120.pdf.

[5] M. Armano et al., Beyond the Required LISA Free-Fall Performance: New LISA Pathfinder Results down to $20 \mu \mathrm{Hz}$, Phys. Rev. Lett. 120, 061101 (2018).

[6] M. Armano et al., Sub-Femto-g Free Fall for Space-Based Gravitational Wave Observatories: LISA Pathfinder Results, Phys. Rev. Lett. 116, 231101 (2016).

[7] B. S. Sheard, G. Heinzel, K. Danzmann, D. A. Shaddock, W. M. Klipstein, and W. M. Folkner, Intersatellite laser ranging instrument for the GRACE follow-on mission, J. Geodes. 86, 1083 (2012).

[8] M. Tinto and J. W. Armstrong, Cancellation of laser noise in an unequal-arm interferometer detector of gravitational radiation, Phys. Rev. D 59, 102003 (1999).

[9] M. Tinto and S. V. Dhurandhar, Time-delay interferometry, Living Rev. Relativity 17, 6 (2014).

[10] B. Sheard, G. Heinzel, and K. Danzmann, LISA long-arm interferometry: An alternative frequency pre-stabilization system, Classical Quantum Gravity 27, 084011 (2010).

[11] O. Gerberding et al., Readout for intersatellite laser interferometry: Measuring low frequency phase fluctuations of high-frequency signals with microradian precision, Rev. Sci. Instrum. 86, 074501 (2015).

[12] S. J. Mitryk, V. Wand, and G. Mueller, Verification of timedelay interferometry techniques using the University of Florida LISA interferometry simulator, Classical Quantum Gravity 27, 084012 (2010).

[13] D. Shaddock, B. Ware, P. G. Halverson, R. E. Spero, and B. Klipstein, Overview of the LISA phasemeter, AIP Conf. Proc. 873, 654 (2006).

[14] G. de Vine, B. Ware, K. McKenzie, R. E. Spero, W. M. Klipstein, and D. A. Shaddock, Experimental Demonstration of Time-Delay Interferometry for the Laser Interferometer Space Antenna, Phys. Rev. Lett. 104, 211103 (2010).

[15] O. Gerberding, B. Sheard, I. Bykov, J. Kullmann, J. J. Esteban Delgado, K. Danzmann, and G. Heinzel, Phasemeter core for intersatellite laser heterodyne interferometry: Modelling, simulations and experiments, Classical Quantum Gravity 30, 235029 (2013).

[16] S. Barke, N. Brause, I. Bykov, J. J. Esteban Delgado, A. Enggaard, O. Gerberding, G. Heinzel, J. Kullmann, S. M. Pedersen, and T. Rasmussen, LISA metrology system final report, Report No. ESA ITT AO/1-6238/10/NL/HB, DTU 
Space, Axcon ApS, AEI Hannover, 2014, http://hdl.handle .net/11858/00-001M-0000-0023-E266-6.

[17] G. Wanner, G. Heinzel, E. Kochkina, C. Mahrdt, B. S. Sheard, S. Schuster, and K. Danzmann, Methods for simulating the readout of lengths and angles in laser interferometers with Gaussian beams, Opt. Commun. 285, 4831 (2012).

[18] E. J. Elliffe, J. Bogenstahl, A. Deshpande, J. Hough, C. Killow, S. Reid, D. Robertson, S. Rowan, H. Ward, and G. Cagnoli, Hydroxide-catalysis bonding for stable optical systems for space, Classical Quantum Gravity 22, S257 (2005).

[19] C. J. Killow, E. D. Fitzsimons, M. Perreur-Lloyd, D. I. Robertson, H. Ward, and J. Bogenstahl, Optical fiber couplers for precision spaceborne metrology, Appl. Opt. 55, 2724 (2016).
[20] T. S. Schwarze, G. Fernández Barranco, D. Penkert, O. Gerberding, G. Heinzel, and K. Danzmann, Optical testbed for the LISA phasemeter, J. Phys. Conf. Ser. 716, 012004 (2016).

[21] M. Tröbs and G. Heinzel, Improved spectrum estimation from digitized time series on a logarithmic frequency axis, Measurement 39, 120 (2006).

[22] M. Tröbs and G. Heinzel, Corrigendum to "Improved spectrum estimation from digitized time series on a logarithmic frequency axis", Measurement 42, 170 (2009).

[23] S. J. Mitryk, G. Mueller, and J. Sanjuan, Hardwarebased demonstration of time-delay interferometry and TDIranging with spacecraft motion effects, Phys. Rev. D 86, 122006 (2012). 